\title{
Completely almost periodic functionals
}

\author{
Volker Runde
}

\begin{abstract}
Using the notion of complete compactness introduced by H. Saar, we define completely almost periodic functionals on completely contractive Banach algebras. We show that, if $(M, \Gamma)$ is a Hopf-von Neumann algebra with $M$ injective, then the space of completely almost periodic functionals on $M_{*}$ is a $C^{*}$-subalgebra of $M$.
\end{abstract}

Keywords: completely compact map; completely almost periodic functional; Hopf-von Neumann algebra.

2000 Mathematics Subject Classification: Primary 47L25; Secondary 22D25, 43A30, 46L07, 47L50.

\section{Introduction}

The almost periodic and weakly almost periodic continuous functions on a locally compact group $G$ form $C^{*}$-subalgebras of $L^{\infty}(G)$, usually denoted by $\mathcal{A P}(G)$ and $\mathcal{W} \mathcal{A P}(G)$, respectively: this is fairly elementary to prove and well known (see [Bur] for instance). In a more abstract setting, one can define, for a general Banach algebra $\mathfrak{A}$ the spaces $\mathcal{A P}(\mathfrak{A})$ and $\mathcal{W} \mathcal{A P}(\mathfrak{A})$ of almost periodic and weakly almost periodic functionals on $\mathfrak{A}$; if $\mathfrak{A}=L^{1}(G)$, we have $\mathcal{A P}(\mathfrak{A})=\mathcal{A P}(G)$ and $\mathcal{W} \mathcal{A} \mathcal{P}(\mathfrak{A})=\mathcal{W} \mathcal{A} \mathcal{P}(G)$.

For $\mathfrak{A}=A(G)$, Eymard's Fourier algebra $($ Eym $)$, the spaces $\mathcal{A P}(\mathfrak{A})$ and $\mathcal{W} \mathcal{A P}(\mathfrak{A})$ are usually denoted by $\mathcal{A P}(\hat{G})$ and $\mathcal{W} \mathcal{A} \mathcal{P}(\hat{G})$ : they were first considered by C. F. Dunkl and D. E. Ramirez ([D-R]) and further studied by E. E. Granirer (Gra 1] and [Gra 2]), A. T.-M. Lau ([Lau $]$, and others. Except in a few special cases, e.g., if $G$ is abelian or discrete and amenable, it is unknown whether $\mathcal{A P}(\hat{G})$ and $\mathcal{W} \mathcal{A P}(\hat{G})$ are $C^{*}$-subalgebras of the group von Neumann algebra $\operatorname{VN}(G)$.

Recently, M. Daws considered the almost periodic and weakly almost periodic functionals on the predual of a Hopf-von Neumann algebra with underlying von Neumann algebra $M([\overline{\mathrm{Daw}}])$. He proved: If $M$ is abelian, then both $\mathcal{A} \mathcal{P}\left(M_{*}\right)$ and $\mathcal{W} \mathcal{A} \mathcal{P}\left(M_{*}\right)$ are $C^{*}$-subalgebras of $M$ ([Daw, Theorems 1 and 4]). Unfortunately, the demand that $M$ be abelian is crucial for Daws' proofs to work (see Run for a discussion).

Over the past two decades, it has become apparent that purely Banach algebraic notions aren't well suited for the study of $A(G)$ : one often has to tweak these notions in a way that takes the canonical operator space structure of $A(G)$-as the predual of the 
group von Neumann algebra - into account. For instance, Banach algebraic amenability of $A(G)$ forces $G$ to be finite-by-abelian $([\mathrm{F}-\mathrm{R}])$ whereas $A(G)$ is operator amenable if and only if $G$ is amenable ([Rua $]$ ), a much more satisfactory result.

We apply this philosophy to almost periodicity. A functional $\phi$ on a Banach algebra $\mathfrak{A}$ is called almost periodic if the maps

$$
\mathfrak{A} \rightarrow \mathfrak{A}^{*}, \quad a \mapsto\left\{\begin{array}{l}
a \cdot \phi \\
\phi \cdot a
\end{array}\right.
$$

are compact. Suppose now that $\mathfrak{A}$ is a completely contractive Banach algebra. Then the maps (因) are completely bounded. There are various definitions that attempt to fit the notion of a compact operator to a completely bounded context (see [Saa, Web], Oik], and [Yew], for instance). We focus on the definition of a completely compact map from Saa, and define $\phi \in \mathfrak{A}^{*}$ to be completely almost periodic if the maps (囵) are completely compact.

Our main result is that, if $(M, \Gamma)$ is a Hopf-von Neumann algebra such that $M$ is injective, then the completely almost periodic functionals on $M_{*}$ form a $C^{*}$-subalgebra of $M$. This applies, in particular, to $A(G)$ in the cases where $G$ is amenable or connected.

\section{Completely compact maps}

There are various ways to adapt the notion of a compact operator to an operator space setting: operator compactness ([Web] and [Yew]), complete compactness ([Saa $)$, and Gelfand complete compactness ( $\underline{\mathrm{Oik}}]$ ).

The notion of a completely compact map between $C^{*}$-algebras was introduced by $\mathrm{H}$. Saar in his Diplomarbeit Saa under G. Wittstock's supervision. The starting point of his definition is the following observation: if $E$ and $F$ are Banach spaces, and $T: E \rightarrow F$ is a bounded linear map, then $T$ is compact if and only if, for each $\epsilon>0$, there is a finite-dimensional subspace $Y_{\epsilon}$ of $F$ such that $\left\|Q_{Y_{\epsilon}} T\right\|<\epsilon$, where $Q_{Y_{\epsilon}}: F \rightarrow F / Y_{\epsilon}$ is the quotient map.

This can be used to define an operator space analog of compactness, namely complete compactness. Saar didn't define complete compactness for maps between general, abstract operator spaces - simply because these objects hadn't been defined yet at that time-, but his definition obviously extends to general operator spaces. (Our reference for operator space theory is $[\mathrm{E}-\mathrm{R}$, the notation and terminology of which we adopt.) In modern language, Saar's definition reads:

Definition 1.1. Let $E$ and $F$ be operator spaces. Then $T \in \mathcal{C B}(E, F)$ is called completely compact if, for each $\epsilon>0$, there is a finite-dimensional subspace $Y_{\epsilon}$ of $F$ such that $\left\|Q_{Y_{\epsilon}} T\right\|_{\mathrm{cb}}<\epsilon$, where $Q_{Y_{\epsilon}}: F \rightarrow F / Y_{\epsilon}$ is the quotient map. 
Remarks. 1. Trivially, completely compact maps are compact.

2. It is obvious that, if $E$ is a Banach space and $F$ is an operator space, then $T \in$ $\mathcal{B}(E, F)=\mathcal{C B}(\max E, F)$ is completely compact if and only if it is compact.

3. On [Saa, pp. 32-34], Saar constructs an example of a compact, completely bounded map on $\mathcal{K}\left(\ell^{2}\right)$ that fails to be completely compact.

4. Complete compactness may not be stable under co-restrictions, i.e., if $T \in \mathcal{C B}(E, F)$ be completely compact, and let $Y$ be a closed subspace of $F$ containing $T E$, then it is not clear why $T$ viewed as an element of $\mathcal{C B}(E, Y)$ should be completely compact.

5. Very recently, complete compactness was put to use for the study of so-called operator multipliers (see $[\mathrm{J}-\mathrm{L}-\mathrm{T}-\mathrm{T}]$ and $[\mathrm{T}-\mathrm{T}]$ ).

Given two operator spaces $E$ and $F$, we write $\mathcal{C K}(E, F)$ for the completely compact operators in $\mathcal{C B}(E, F)$.

The following proposition is essentially [Saa, Lemma 1 a) and Lemma 2]. (Of course, Saar only considers maps between $C^{*}$-algebras, but his proofs carry over more or less verbatim.)

Proposition 1.2. Let $E$ and $F$ be operator spaces. Then:

(i) $\mathcal{C K}(E, F)$ is a closed subspace of $\mathcal{C B}(E, F)$ containing all finite rank operators;

(ii) if $T \in \mathcal{C K}(E, F), X$ is another operator space, and $R \in \mathcal{C B}(X, E)$, then $T R \in$ $\mathcal{C K}(X, F)$

(iii) if $T \in \mathcal{C K}(E, F), Y$ is another operator space, and $S \in \mathcal{C B}(F, Y)$, then $S T \in$ $\mathcal{C K}(E, Y)$.

From Schauder's theorem and Saar's characterization of compact operators, it follows immediately that a bounded linear operator $T$ from a Banach space $E$ into a Banach space $F$ is compact if and only if, for each $\epsilon>0$, there is a closed subspace $X_{\epsilon}$ of $E$ with finite co-dimension such that $\left\|\left.T\right|_{X_{\epsilon}}\right\|<\epsilon$ (compare also [Lac]).

Following $\mathrm{Oik}$, we define:

Definition 1.3. Let $E$ and $F$ be operator spaces. Then $T \in \mathcal{C B}(E, F)$ is called Gelfand completely compact if, for each $\epsilon>0$, there is a closed subspace $X_{\epsilon}$ of $E$ with finite co-dimension such that $\left\|\left.T\right|_{X_{\epsilon}}\right\|_{\mathrm{cb}}<\epsilon$.

Remarks. 1. Obviously, $T \in \mathcal{C B}(E, F)$ is Gelfand completely compact if and only if $T^{*} \in \mathcal{C B}\left(F^{*}, E^{*}\right)$ is completely compact (and vice versa).

2. A result analogous to Proposition 1.2 holds for Gelfand completely compact maps. 
3. Based on results from the unpublished paper [Web], T. Oikhberg gives examples of completely compact maps that fail to be Gelfand completely compact and vice versa ([Oik, pp. 155-156]). Hence, an analog for Schauder's theorem fails for complete compactness.

Under certain circumstances, every completely compact map is Gelfand completely compact ([ik, Theorem 3.1]). For our purposes, the following is important (see [E-R, p. 70] for the notion of an injective operator space):

Proposition 1.4. Let $E$ and $F$ be operator spaces such that $E^{*}$ and $F^{*}$ are injective. Then the following are equivalent for $T \in \mathcal{C B}\left(E, F^{*}\right)$ :

(i) $T$ is completely compact;

(ii) $T$ is Gelfand completely compact;

(iii) $T$ is a cb-norm limit of finite rank operators.

Proof. Obviously, (iii) implies both (i) and (ii).

(ii) $\Longrightarrow$ (iii): Let $T$ be Gelfand completely compact, and let $\epsilon>0$. Then there is a closed subspace $X_{\epsilon}$ of $E$ with finite co-dimension such that $\left\|\left.T\right|_{X_{\epsilon}}\right\|_{\mathrm{cb}}<\epsilon$. Due to the injectivity of $F^{*}$, there is $\tilde{T} \in \mathcal{C B}\left(E, F^{*}\right)$ such that $\left.\tilde{T}\right|_{X_{\epsilon}}=\left.T\right|_{X_{\epsilon}}$ and $\|\tilde{T}\|_{\mathrm{cb}}=\left\|\left.T\right|_{X_{\epsilon}}\right\|_{\mathrm{cb}}<\epsilon$. Then $S:=T-\tilde{T}$ satisfies $\|S-T\|_{\mathrm{cb}}<\epsilon$ and vanishes on $X_{\epsilon}$; since $X_{\epsilon}$ has finite codimension, $S$ must have finite rank..

(i) $\Longrightarrow$ (iii): As $T^{*} \in \mathcal{C B}\left(F^{* *}, E^{*}\right)$ is Gelfand completely compact, it is a cb-norm limit of finite rank operators - by the argument used for (ii) $\Longrightarrow$ (iii) - as is its adjoint $T^{* *} \in \mathcal{C B}\left(E^{* *}, F^{* * *}\right)$. Hence, given $\epsilon>0$, there is a finite rank operator $S: E^{* *} \rightarrow F^{* * *}$ such that $\left\|S-T^{* *}\right\|_{\mathrm{cb}}<\epsilon$. Let $Q: F^{* * *} \rightarrow F^{*}$ be the Dixmier projection, i.e., the adjoint of the canonical embedding of $F$ into $F^{* *}$. Then $S_{0}:=\left.Q S\right|_{E}$ is a finite rank operator from $E$ to $F^{*}$ such that $\left\|S_{0}-T\right\|_{\mathrm{cb}}<\epsilon$.

\section{Completely almost periodic functionals}

If $\mathfrak{A}$ is a Banach algebra, then its dual spaces becomes a Banach $\mathfrak{A}$-bimodule via

$$
\langle x, a \cdot \phi\rangle:=\langle x a, \phi\rangle \quad \text { and } \quad\langle x, \phi \cdot a\rangle:=\langle a x, \phi\rangle \quad\left(a, x \in \mathfrak{A}, \phi \in \mathfrak{A}^{*}\right) .
$$

For $\phi \in \mathfrak{A}^{*}$, define $L_{\phi}, R_{\phi}: \mathfrak{A} \rightarrow \mathfrak{A}^{*}$ via

$$
L_{\phi} a:=\phi \cdot a \quad \text { and } \quad R_{\phi} a:=a \cdot \phi \quad(a \in \mathfrak{A}) .
$$

A functional $\phi \in \mathfrak{A}^{*}$ is commonly called almost periodic if $L_{\phi}$ and $R_{\phi}$ are compact operators. (As $L_{\phi}=\left.R_{\phi}^{*}\right|_{\mathfrak{A}}$ and $R_{\phi}=\left.L_{\phi}^{*}\right|_{\mathfrak{A}}$, it is sufficient to require that only one of $L_{\phi}$ and $R_{\phi}$ be compact.) We denote the space of all almost periodic functionals in $\mathfrak{A}^{*}$ by $\mathcal{A} \mathcal{P}(\mathfrak{A})$. 
An operator space that is also an algebra such that multiplication is completely contractive, is called a completely contractive Banach algebra.

We define:

Definition 2.1. Let $\mathfrak{A}$ be a completely contractive Banach algebra. We call $\phi \in \mathfrak{A}^{*}$ completely almost periodic if $L_{\phi}, R_{\phi} \in \mathcal{C K}\left(\mathfrak{A}, \mathfrak{A}^{*}\right)$ and denote the collection of completely almost periodic functionals in $\mathfrak{A}^{*}$ by $\mathcal{C} \mathcal{A} \mathcal{P}(\mathfrak{A})$.

Remarks. $\quad$ 1. Obviously, $\mathcal{C} \mathcal{A} \mathcal{P}(\mathfrak{A})$ is a closed linear subspace of $\mathfrak{A}^{*}$.

2. Since Schauder's theorem is no longer true for complete compactness, we do need the requirement that both $L_{\phi}$ and $R_{\phi}$ are completely compact.

3. Since $L_{\phi}=\left.R_{\phi}^{*}\right|_{\mathfrak{A}}$ and $R_{\phi}=\left.L_{\phi}^{*}\right|_{\mathfrak{A}}$, we could have replaced in complete almost periodicity - based on complete compactness - in Definition 2.1 by the analogous notion involving Gelfand complete compactness instead: we would still have obtained the same functionals.

We shall now look at a special class of completely contractive Banach algebras which arise naturally in abstract harmonic analysis.

Recall that a Hopf-von Neumann algebra is a pair $(M, \Gamma)$ where $M$ is a von Neumann algebra and $\Gamma: M \rightarrow M \bar{\otimes} M$ is a co-multiplication, i.e., a normal, unital, ${ }^{*}$-homomorphism satisfying

$$
(\mathrm{id} \otimes \Gamma) \circ \Gamma=(\Gamma \otimes \mathrm{id}) \circ \Gamma .
$$

The co-multiplication induces a product $*$ on $M_{*}$ via

$$
\langle f * g, x\rangle:=\langle f \otimes g, \Gamma x\rangle \quad\left(f, g \in M_{*}, x \in M\right),
$$

which turns $M_{*}$ into a completely contractive Banach algebra.

Examples. 1. Let $G$ be a locally compact group, and define a co-multiplication $\Gamma$ : $L^{\infty}(G) \rightarrow L^{\infty}(G) \bar{\otimes} L^{\infty}(G)$ - noting that $L^{\infty}(G) \bar{\otimes} L^{\infty}(G) \cong L^{\infty}(G \times G)$-by letting

$$
(\Gamma \phi)(x, y):=\phi(x y) \quad\left(x, y \in G, \phi \in L^{\infty}(G)\right) .
$$

Then $L^{\infty}(G)_{*}=L^{1}(G)$ with the product induced by $\Gamma$ is just the usual convolution algebra $L^{1}(G)$. Since the operator space structure on $L^{1}(G)$ is maximal, $\mathcal{C} \mathcal{A P}\left(L^{1}(G)\right)$ equals $\mathcal{A P}\left(L^{1}(G)\right)$; it consists precisely of the almost periodic continuous functions on $G$ in the classical sense (see [Bur], for instance) and thus, in particular, is a $C^{*}$-subalgebra of $L^{\infty}(G)$.

2. Let again $G$ be a locally compact group, let $\operatorname{VN}(G)$ be its group von Neumann algebra, i.e., the von Neumann algebra generated by $\lambda(G)$, where $\lambda$ is the left regular representation of $G$ on $L^{2}(G)$, and let $\Gamma: \mathrm{VN}(G) \rightarrow \mathrm{VN}(G) \bar{\otimes} \mathrm{VN}(G)$ be given by

$$
\Gamma \lambda(x)=\lambda(x) \otimes \lambda(x) \quad(x \in G) .
$$


Then $\mathrm{VN}(G)_{*}$ is Eymard's Fourier algebra $A(G)$ (Eym), and the product induced by $\Gamma$ is pointwise multiplication. The space $\mathcal{A P}(A(G))$ —often denoted by $\mathcal{A P}(\hat{G})$ was first considered in [D-R] and further studied in [Gra 1] and [Lau], for instance.

Except in a few special cases, e.g., if $G$ is abelian or discrete and amenable (as a consequence of [Gra 1, Theorem 12] and Gra 2, Proposition 2]), is unknown whether or not $\mathcal{A P}(\hat{G})$ is a $C^{*}$-subalgebra of $\operatorname{VN}(G)$.

The picture changes once we replace almost periodicity with complete almost periodicity:

Theorem 2.2. Let $(M, \Gamma)$ be a Hopf-von Neumann algebra such that $M$ is injective. Then $\mathcal{C} \mathcal{A P}\left(M_{*}\right)$ is a $C^{*}$-subalgebra of $M$.

Proof. Let $x \in M$. Then, by Proposition 1.4, $L_{x}$ is completely compact if and only if it is a cb-norm limit of finite rank operators. In view of the completely isometric identifications ([E-R, Corollary 7.1.5] and [E-R, Theorem 7.2.4])

$$
M \bar{\otimes} M \cong\left(M_{*} \hat{\otimes} M_{*}\right)^{*} \cong \mathcal{C B}\left(M_{*}, M\right)
$$

and of [E-R, Proposition 8.1.2], this means that $L_{x}$ is completely compact if and only if $\Gamma x \in M \check{\otimes} M$. A similar assertion holds for $R_{x}$.

All in all, we have that

$$
\mathcal{C} \mathcal{A P}\left(M_{*}\right)=\{x \in M: \Gamma x \in M \check{\otimes} M\} .
$$

Since $M \check{\otimes} M$ is nothing but the spatial $C^{*}$-tensor product of $M$ with itselt and thus $C^{*}$-subalgebra of $M \bar{\otimes} M$, and since $\Gamma$ is a ${ }^{*}$-homomorphism, this proves the claim.

If $G$ is an amenable or connected locally compact group, then $\operatorname{VN}(G)$ is well known to be injective. Writing $\mathcal{C} \mathcal{A} \mathcal{P}(\hat{G})$ for $\mathcal{C} \mathcal{A} \mathcal{P}(A(G))$, we thus have:

Corollary 2.3. Let $G$ be an amenable or connected locally compact group. Then $\mathcal{C} \mathcal{A P}(\hat{G})$ is a $C^{*}$-subalgebra of $\mathrm{VN}(G)$.

Remarks. 1. If $G$ is abelian - or, more generally, finite-by-abelian - , then the canonical operator space structure on $A(G)$ is equivalent to $\max A(G)$, so that $\mathcal{C} \mathcal{A} \mathcal{P}(\hat{G})=$ $\mathcal{A P}(\hat{G})$.

2. Suppose that $G$ is discrete and amenable. Then $\mathcal{A P}(\hat{G})$ equals $C_{r}^{*}(G)$, the (reduced) group $C^{*}$-algebra of $G$ by [Gra 2, Proposition 3(b)]. Since $\Gamma C_{r}^{*}(G) \subset$ $C_{r}^{*}(G) \check{\otimes} C_{r}^{*}(G) \subset \mathrm{VN}(G) \check{\otimes} \mathrm{VN}(G)$, an inspection of the proof of Theorem 2.2 shows that therefore $\mathcal{A P}(\hat{G}) \subset \mathcal{C} \mathcal{A} \mathcal{P}(\hat{G})$ and thus, trivially, $\mathcal{A P}(\hat{G})=\mathcal{C} \mathcal{A P}(\hat{G})$. 


\section{References}

[Bur] R. B. Burckel, Weakly Almost Periodic Functions on Semigroups. Gordon and Breach, 1970.

[Daw] M. DAws, Weakly almost periodic functionals on the measure algebra. Math. Z. 265 (2010), 285-296. .

[D-R] C. F. DunkL and D. E. RamiRez, Weakly almost periodic functionals on the Fourier algebra. Trans. Amer. Math. Soc. 185 (1973), 501-514.

[E-R] E. G. Effros and Z.-J. Ruan, Operator Spaces. London Mathematical Society Monographs (New Series) 25, Clarendon Press, 2000.

[Eym] P. Eymard, L'algèbre de Fourier d'un groupe localement compact. Bull. Soc. Math. France 92 (1964), 181-236.

[F-R] B. E. Forrest and V. Runde, Amenability and weak amenability of the Fourier algebra. Math. Z. 250 (2005), 731-744.

[Gra 1] E. E. Granirer, Weakly almost periodic and uniformly continuous functionals on the Fourier algebr of any locally compact group. Trans. Amer. Math. Soc. 189 (1974), $371-382$.

[Gra 2] E. E. Granirer, Density theorems for some linear subspaces and some $C^{*}$ subalgebras of VN $(G)$. In: Symposia Mathematica XXII, pp. 61-70, Academic Press, London-New York, 1977.

[J-L-T-T] K. Juschenko, R. H. Levene, I. G. Todorov, and L. Turowska, Compactness properties of operator multipliers. J. Funct. Anal. 256 (2009), 3772-3805.

[Lac] H. E. LACEY, Generalizations of Compact Operators in Locally Convex Topological Linear Spaces. Ph.D. Thesis, New Mexico State University, 1963.

[Lau $\quad$ A. T.-M. LAU, Uniformly continuous functionals on the Fourier algebr of any locally compact group. Trans. Amer. Math. Soc. 251 (1974), 371-382.

[Oik] T. OikhBerg, Direct sums of operator spaces. J. London Math. Soc. (2) 64 (2001), 144-160.

[Pal] T. W. Palmer, Banach Algebras and the General Theory of *-Algebras. Volume I: Algebras and Banach Algebras. Ecyclopedia of Mathematics and its Applications 49, Cambridge University Press, 1994.

[Rua] Z.-J. Ruan, The operator amenability of $A(G)$. Amer. J. Math. 117 (1995), 14491474 .

[Run] V. Runde, Co-representations of Hopf-von Neumann algebras on operator spaces other than column Hilbert space. Bull. Aust. Math. Soc. 82 (2010), 205-210.

[Saa] H. SAAR, Kompakte, vollständig beschränkte Abbildungen mit Werten in einer nuklearen $C^{*}$-Algebra. Diplomarbeit, Universität des Saarlandes, 1982.

[T-T] I. G. Todorov and L. Turowska, Schur and operator multipliers. In: R. J. Loy, V. Runde, and A. SoŁtysiak (eds.), Banach Algebras 2009. Banach Center Publications, to appear. 
[Web] C. WebsteR, Matrix compact sets and operator approximation properties. ArXiv: math/9894093.

[Yew] K. L. YEw, Compactness and approximation property with respect to an operator space. Indiana Univ. Math. J. 56 (2007), 3075-3128.

[July 1, 2018]

Author's address: Department of Mathematical and Statistical Sciences

University of Alberta

Edmonton, Alberta

Canada T6G 2G1

E-mail: $\quad$ vrunde@ualberta.ca

URL: $\quad$ http://www.math.ualberta.ca/ runde/ 